\title{
Interstitial Lung Disease as Presenting Featurie of SLE
}

\section{Sumantro Mondal, Tony Ełe, Debanjali Sinha, Atanu Chakraborty, Arifit Nag,} Soumik Sarkar, Jyotirmoy Pal, Alakendu Ghosh

From the Department of Medicine, Institute of Post Graduate Medical Education and Research, Kolkata, India.

\begin{abstract}
:
Interstitial lung disease (ILD) has been considered as an unusual pulmonary manifestation of systemic lupus erythematosus. Patients of ILD usually present with progressive dry cough and exertional dyspnea. ILD is usually seen in long standing SLE patients and follows a chronic course. ILD as the initial manifestation of SLE is very rare. Our middle aged female patient presented with pulmonary symptoms, renal impairment and arthralgia. She was finally diagnosed as having SLE by relevant autoantibody profile and renal biopsy along with HRCT proven ILD.
\end{abstract}

Key words: Lupus erythematosus, Systemic, Lung diseases, Interstitial, Dyspnea, Cough, Arthalgia.

\section{Introduction}

Systemic lupus erythematosus is a multisystem autoimmune disorder with varied clinical presentations and wide spectrum of organ involvement. Females of reproductive age group are more commonly affected by the disease though age range can vary. Study from a tertiary care hospital from Eastern India showed female:male ratio as high as 39:1 [1]. The disease usually presents with cutaneous, musculo-skeletal, renal, neurologic, hematological and pulmonary manifestations. Pulmonary manifestations usually follow a chronic course except for diffuse alveolar hemorrhage and acute lupus pneumonitis which follow an acute course. Pleurisy or pleural effusion is the most common pulmonary manifestations of SLE [2,3]. Interstitial lung disease (ILD), pulmonary embolism, pulmonary vasculitis and hypertension, bronchiolitis obliterans with or without organizing pneumonia (BOOP), shrinking lung syndrome, opportunistic pulmonary infections and toxicity due to immunosuppressive drugs are the other pulmonary complications of SLE $[4,5]$. ILD is not so common for SLE and even much rarer to be the initial manifestation of SLE. We describe a patient of SLE in whom ILD was one of the presenting features of the disease along with renal and musculo-skeletal involvement.

\section{Case Report}

A 40 year old non-diabetic, non-hypertensive female presented with bipedal swelling, puffiness of face for one month and oliguria for ten days. The

Corresponding Author: Dr. Sumantro Mondal

Email: drmsumantro@gmail.com

Received: November 7, 2013 | Accepted: February 5, 2014 | Published Online: March 5, 2014

This is an Open Access article distributed under the terms of the Creative Commons Attribution License (creativecommons.org/licenses/by/3.0)

Conflict of interest: None declared | Source of funding: Nil | DOl: http://dx.doi.org/10.17659/01.2014.0021 
patient was apparently well one month back when she started passing frothy urine. She had a history of dry cough for the last three years along with exertional dyspnea for the last two years. There was a history of arthralgia involving large and small joints of all four limbs for the last six months. There was no history of orthopnoea, paroxysmal nocturnal dyspnea, fever, rash, photosensitivity and oral ulceration. General examination revealed pallor and bipedal pitting edema. Her pulse rate was 96/minute, blood pressure 146/90 mm Hg. Respiratory system examination revealed bilateral fine end inspiratory crepitations, predominantly at lung bases with shrinkage of lung volume. Other systems were normal.

Hematological and biochemical investigations revealed $\mathrm{Hb}: 9.6 \mathrm{gm} / \mathrm{dL}$, total leucocyte count:5100/cumm, platelet count: 1.6 lakh/cumm, serum urea: $124 \mathrm{mg} / \mathrm{dL}$ and creatinine: $3.8 \mathrm{mg} /$ dL. Liver function test was normal. Urine routine examination revealed $3+$ albumin, WBC casts, and granular casts. Twenty four hour urinary protein excretion was $1.9 \mathrm{gm}$ (normal value $<150 \mathrm{mg} / 24$

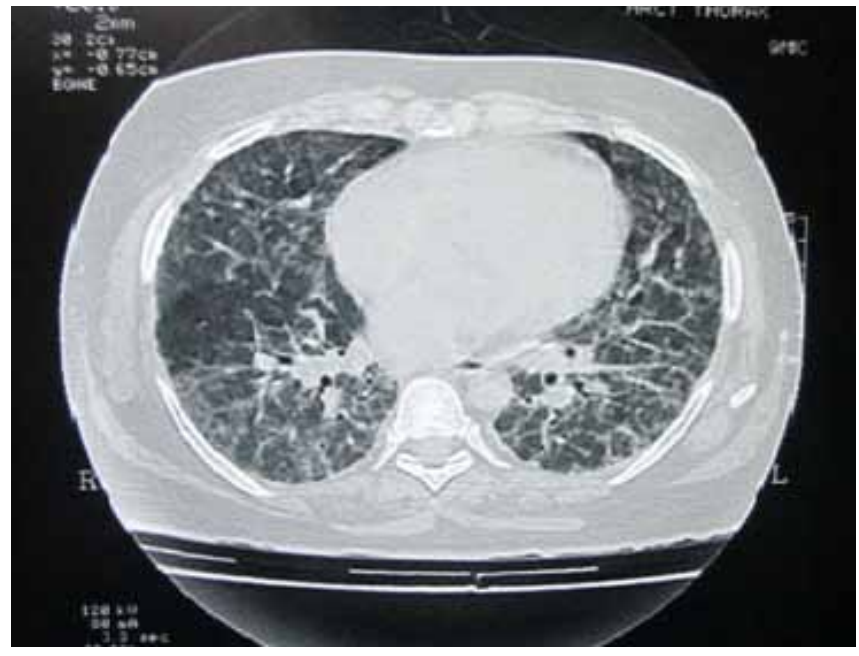

Fig.1: HRCT scan of thorax showing diffuse bilateral ground glass opacification, interlobular septal thickening and subtle bronchiectatic changes in basal lung fields, suggestive of ILD. hours). Chest X-ray was unremarkable. Blood for ANA by Hep 2 method was positive $(4+, 1: 100$ dilution), anti dsDNA-345 U/mL (normal range: $<100 \mathrm{lU} / \mathrm{mL}$ ). Blood for C3 and C4 were $54 \mathrm{mg} /$ $\mathrm{dL}$ (normal range: $90-180 \mathrm{mg} / \mathrm{dL}$ ) and $24 \mathrm{mg} /$ $\mathrm{dL}$ (normal range: $10-40 \mathrm{mg} / \mathrm{dL}$ ) respectively. Ultrasonography of whole abdomen was normal. HRCT thorax revealed diffuse bilateral ground glass opacification, interlobular septal thickening and subtle bronchiectatic changes in basal lung fields, suggestive of interstitial lung disease [Fig.1]. Pulmonary function test showed restrictive pattern of lung disease compatible with interstitial lung disease. Renal biopsy report showed $\lg G, \lg A$, $\mathrm{C} 3$ and $\mathrm{Clq}$ deposition along the capillary walls, consistent with membranous lupus nephritis (lupus nephritis class V) [Fig.2]. A final diagnosis of SLE was made and the patient was started withhydroxylchloroquine sulfate and immunosupression with advice for regular follow up.

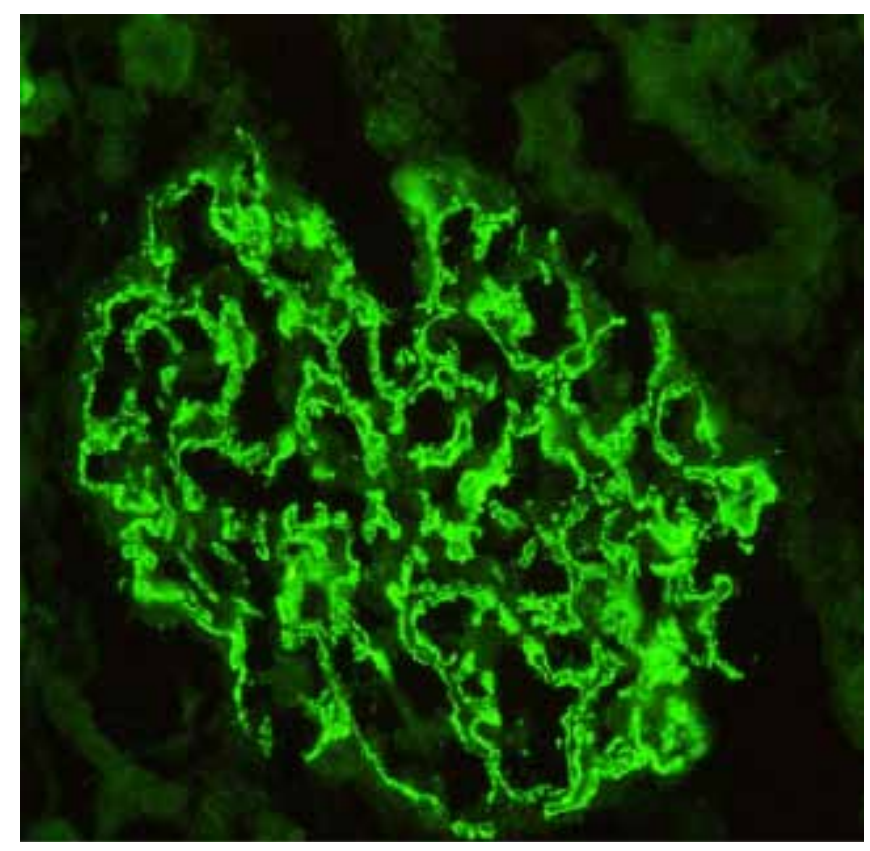

Fig.2: Renal biopsy specimen with immunofluorescence staining showing deposition of $\lg G, \lg A, C 3$ and $C l q$ along the capillary walls. 


\section{Discussion}

Mild lung disease is present in about one third of SLE patients but clinically significant disease is seen in only $3-8 \%$ of the lupus population [6]. ILD are a heterogenous group of disorders characterized by varying degree inflammation and fibrosis of the lung parenchymal tissue. ILD is seen in about $10 \%$ of lupus cases $[1,7]$. Non-specific interstitial pneumonia (NSIP) is the most commonly observed histopathological pattern of ILD in SLE, but other patterns can also be present. It is important to note that unlike some other connective tissue diseases, ILD in SLE usually follows a benign course with a subacute to chronic presentation during the disease process [1]. ILD as the presenting feature of SLE is rare with few case reports in literature $[8,9]$. Kumar A et al. reported a case of 40 year old Asian female SLE patient in whom progressive dyspnea was the initial manifestation [8]. Contrary to our patient dry cough, which is a classical feature of ILD was absent in their patient. There was possibility of co-existent COPD in their case, evidenced by hyperinflation of lung fields due to long term bio-mass exposure. Similar to our patient, they confirmed the diagnosis of SLE by serum autoantibody profile though no information about renal biopsy result is available from their report. We performed renal biopsy in our patient as she fulfilled the indications for the same and also for determining treatment protocol. Esmaeilbeigi $\mathrm{F}$ et al. reported another case of SLE with desquamative interstitial pneumonia (DIP), a rare form of ILD [9]. In this case, the diagnosis of SLE was made long before the development of DIP, so it could not regarded as the presenting feature of SLE. In our patient renal and articular manifestations were also present along with ILD that helped us for consideration of SLE as a possible diagnosis which was finally confirmed by renal biopsy.

\section{Conclusion}

ILD may be the initial manifestation of SLE. SLE should be a diagnostic consideration in patient with ILD, especially in female of reproductive age group with other systemic involvement.

\section{References}

1. Ghosh A, Das T, Ghosh A, Karmakar P, Pal J. Evaluation of respiratory manifestations in systemic lupus erythematosus with special reference to pulmonary interstitial involvement. J Indian Med Assoc. 2012;1 10(2):109-111.

2. Peter HS, Paul FD. Pulmonary manifestations of systemic lupus erythematosus in adults. 2010. Available from: URL:http://www.UpToDate. com. V19.3. Accessed on 5th Feb.2014.

3. Fekih L, Boussoffara L, Chaouachi S, Fenniche $\mathrm{S}$, Abdelghaffar H, Akrout I, et al. Thoracic manifestations of systemic lupus erythematosus. Tunis Med. 2011 ;89(3):269-273.

4. Keane MP, Lynch JP $3^{\text {rd }}$. Pleuro-pulmonary manifestations of systemic lupus erythematosus. Thorax. 2000;55(2):159-166.

5. Kakati S, Doley B, Pal S, Deka UJ. Pulmonary manifestations in systemic lupus erythematosus (SLE) with special reference to HR CT. J Assoc Physicians India. 2007;55:839-834.

6. Sant SM, Doran M, Fenelon HM, Breatnach ES. Pleuro-pulmonary abnormalities in patients with systemic lupus erythematosus: assessment with high resolution computed tomography, chest radiography and pulmonary function tests. Clin Exp Rheumatol. 1997; 15:507-513.

7. Torre O, Harari S. Pleural and pulmonary involvement in systemic lupus erythematosus. Presse Med. 2011 ;40:e19-29.

8. Kumar A, Khan U, Shrestha B, Thapa S, Rizvi N. Interstitial lung disease as initial manifestation of systemic lupus erythematosus. J Nepal Health Res Counc. 2013;1 1(23):83-85.

9. Esmaeilbeigi F, Juvet S, Hwang D, Mittoo S. Desquamative interstitial pneumonitis in a patient with systemic lupus erythematosus. Can Respir J. 2012;19(1):50-52. 